\title{
Observation of Unidirectional Solitonlike Edge States in Nonlinear Floquet Topological Insulators
}

\author{
Sebabrata Mukherjee $\odot^{1,2, *}$ and Mikael C. Rechtsman $\odot^{1, \dagger}$ \\ ${ }^{1}$ Department of Physics, The Pennsylvania State University, University Park, Pennsylvania 16802, USA \\ ${ }^{2}$ Department of Physics, Indian Institute of Science, Bangalore 560012, India
}

(Received 4 May 2021; revised 18 October 2021; accepted 21 October 2021; published 22 December 2021)

\begin{abstract}
A salient feature of solid-state quantum-Hall-type topological materials in two dimensions is the presence of conducting electronic edge states that are insensitive to scattering by disorder. Such unidirectional edge states have been predicted and observed in many other experimental settings, including photonics, mechanical, and acoustic structures. It is of great interest to understand how topological states behave in the presence of interparticle interactions and nonlinearity. Here, we experimentally demonstrate backscatterimmune unidirectional solitonlike nonlinear states on the edge of photonic topological insulators consisting of laser-written waveguides. As a result of the optical Kerr nonlinearity of the ambient glass, the solitonlike wave packet forms a long-lived quasilocalized coherent structure that slowly radiates power into the bulk and along the edge. The realization of solitonlike edge states paves the way to an understanding of nonlinear and interacting topological systems.
\end{abstract}

DOI: $10.1103 /$ PhysRevX.11.041057

Subject Areas: Nonlinear Dynamics, Optics

Topological Insulators

\section{INTRODUCTION}

The field of topological photonics [1-5] shows great promise for the discovery of new fundamental science and its implications for advances in optical devices [6-9]. Quantum-Hall-like topological states [10-15] for electromagnetic waves were first proposed [1] in the context of photonic crystals and were experimentally demonstrated [2] using magneto-optical materials at microwave frequencies. The concept of "Floquet topological insulators"namely, inducing topologically nontrivial behavior using dynamical modulation - is used in waveguide arrays for the realization of optical chiral edge states in Chern [3] and anomalous Floquet topological insulators [16-19]. In these systems, the component waveguides are spatially modulated along the propagation axis to effectively break timereversal symmetry in the transverse plane and, thus, enable the presence of topological gaps and chiral edge states. Analogous techniques are used to realize topological states in many other experimental platforms, such as ultracold atoms [20,21], mechanical [22,23] circuits [24], and

\footnotetext{
*mukherjee@iisc.ac.in

†mcrworld@psu.edu
}

Published by the American Physical Society under the terms of the Creative Commons Attribution 4.0 International license. Further distribution of this work must maintain attribution to the author(s) and the published article's title, journal citation, and DOI. exciton polaritons [25-27], and also in the context of equatorial waves [28].

Solitons are nonlinear wave packets that balance nonlinearity with the tendency to spread due to diffraction or dispersion. The result is a wave function that maintains its shape as it propagates. Solitons play a central role in the theory of nonlinear differential equations as the solutions that form the basis for the inverse scattering transformation $[29,30]$ and arise naturally in nonlinear and interacting systems such as water waves [31], photonic systems [32-36], and Bose-Einstein condensates [37,38]. Their ubiquity across different physical platforms speaks to the generality of the equations describing interactions in bosonic systems: The nonlinear diffraction of light and the temporal dynamics of a dense Bose-Einstein condensate are both described by the nonlinear Schrödinger equation (also called the Gross-Pitaevskii equation). The question of how solitons behave in topological systems, both within the bulk and localized to the edge, is an active and open one [39-48].

Here, we observe unidirectional solitonlike wave packets on the edge of a two-dimensional anomalous photonic Floquet topological insulator. We also demonstrate that this nonlinear state can propagate around a corner of the lattice without backscattering. By solitonlike wave packet, we mean a nonlinear quasilocalized solution with a finite lifetime. The reason that they must be called "solitonlike" is that they radiate power into the bulk and along the edge at a small but nonzero rate; see Fig. 1(a). Indeed, because of the discreteness of the system, a nonlinear wave packet experiences a nonzero Peierls-Nabarro barrier [49], which 

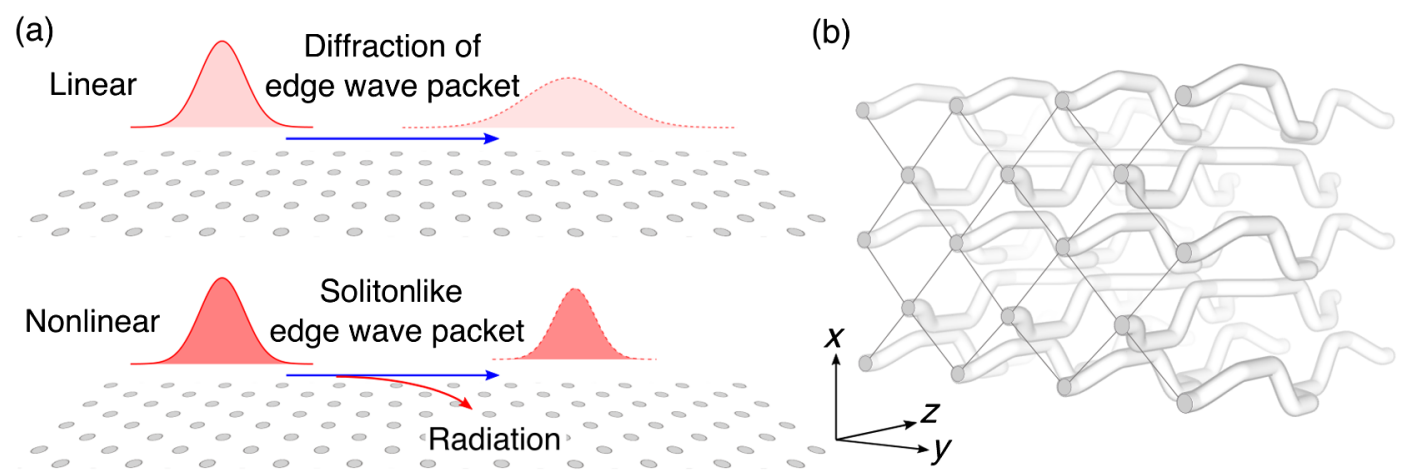

(c)

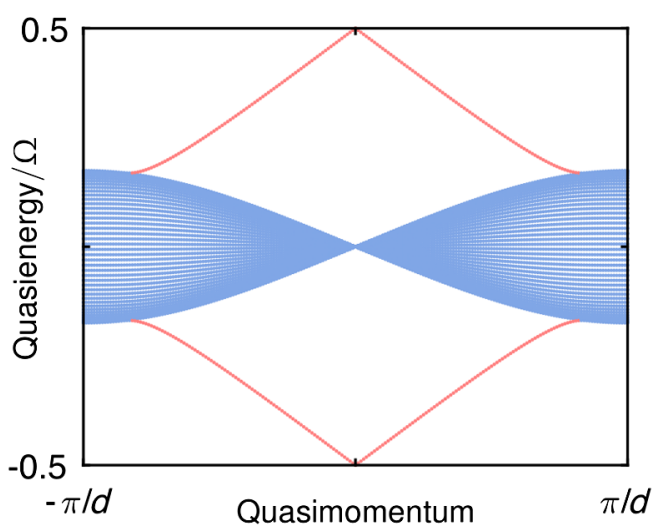

(d)

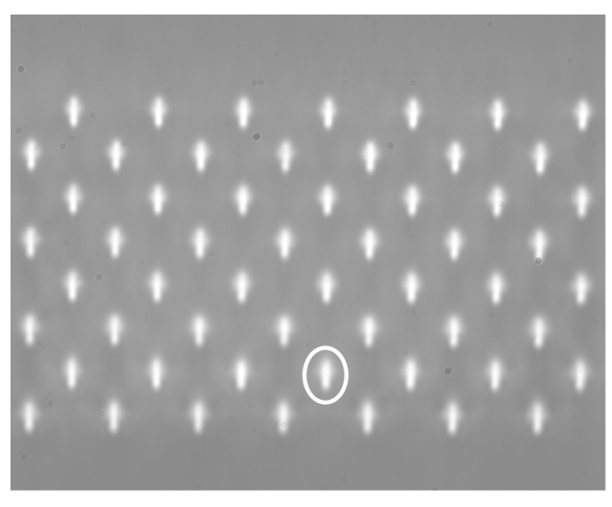

FIG. 1. Implementation of solitonlike edge states. (a) Unidirectional propagation of a wave packet along a linear topological insulator-because of the group velocity dispersion of the edge states, the edge wave packet diffracts (upper plot). The linear diffraction of the edge wave packet can be almost balanced by nonlinearity that arises from optical Kerr nonlinearity. Because of the discreteness, the solitonlike edge state radiates power along the edge and into the bulk at a finite and controllable rate (lower plot). (b) Schematic depiction of the implementation of a photonic Floquet topological insulator using a three-dimensional waveguide array. (c) Quasienergy spectrum of the bipartite photonic lattice $(\delta=0.18)$ showing two ungapped bulk bands (blue) with zero net Chern number and chiral edge states (red). (d) Micrograph of the facet of a periodically driven square lattice consisting of 56 waveguides. A single-site input state, launched at the circled site on the zigzag edge, has a significant overlap with the topological edge states.

causes the radiation of energy to the ambient extended modes [50-52].

The paper is organized as follows. In Sec. II, we discuss the driving protocol, quasienergy spectrum, and topological characteristics of an effectively dimerized anomalous Floquet topological insulator. In Sec. III, we study the nonlinear dynamics through the periodically modulated topological lattices and discuss the experimental signatures of the unidirectional solitonlike edge states. Then, in Sec. IV, we present the experimental results on the observation of solitonlike edge states traveling along an edge and around a corner of the waveguide lattices. Finally, in Sec. V, we highlight more about the significance of this work and its future implications.

\section{NONLINEAR FLOQUET TOPOLOGICAL INSULATOR}

We utilize a square array of optical waveguides [Fig. 1(b)], with periodically modulated nearest-neighbor evanescent couplings, where the Kerr nonlinearity increases the refractive index in proportion to the local intensity of light.
Through this waveguide lattice, the scalar-paraxial propagation of light is governed by the following discrete nonlinear Schrödinger equation:

$$
i \frac{\partial}{\partial z} \Phi_{s}(z)=\sum_{\left\langle s^{\prime}\right\rangle}-J_{m}(z) \Phi_{s^{\prime}}-\left|\Phi_{s}\right|^{2} \Phi_{s}
$$

where the propagation distance $z$ plays the role of time, $s$ labels the lattice sites, the summation is taken over nearestneighbor sites, and $J_{m}(z)[m=1, \ldots, 4]$ denote the couplings at the $m$ th step. Here, the complete modulation cycle is divided into four steps. For the $m$ th driving step, we define $\Lambda_{m}=\int d z J_{m}(z)$, which determines the transfer of optical power from a given site to one of its nearest neighbors in the linear regime. All four couplings $J_{m}(z)$ resemble a steplike function with a fixed gradual rise and fall time; however, the driving steps are engineered in a bipartite fashion such that $\Lambda_{1}=\Lambda_{3}=\pi / 2(1+\delta)$ and $\Lambda_{2}=\Lambda_{4}=\pi / 2(1-\delta)$-see the Appendix A. The lattice can, thus, be considered to be effectively "dimerized" with $\delta$ as the degree of dimerization. The localization peak caused by this dimerization 
unambiguously proves the existence of the solitonlike edge states in our experiments, as discussed later in Secs. III and IV.

The linear tight-binding Hamiltonian associated with this model changes periodically in $z, H\left(z+z_{0}\right)=H(z)$, with a period $z_{0}=2 \pi / \Omega$. Note that the driving frequency $\Omega$ remains unaltered when $\delta$ is varied. The quasienergy spectrum of this $z$-periodic lattice system can be obtained by diagonalizing the propagator over one complete period, where the propagator is given by $\hat{U}\left(z_{0}\right)=$ $\mathcal{T} \exp \left[-i \int_{0}^{z_{0}} d \tilde{z} \hat{H}(\tilde{z})\right]$, where $\mathcal{T}$ indicates the "time" ordering in $\tilde{z}$. Figure 1 (c) shows the quasienergy spectrum for $\delta=0.18$, calculated using a strip geometry aligned along the vertical direction and periodic along the horizontal direction. The spectrum consists of two ungapped bulk bands (henceforth referred to as the bulk band) and one chiral edge state (per edge) connecting the top and bottom of the bulk band.

The topological nature of the system, as well as the width of the bulk band, can be controlled by $\delta$. For $\delta=0$, the bulk band is perfectly dispersionless (flat) and the system is topological; the bulk bandwidth increases with $\delta$, and the band gap closes at $\delta=0.5$-see Appendix A. Therefore, unidirectional propagating topological edge states exist for $0 \leq \delta<0.5$. The topology of such a periodically driven model is governed by the Floquet winding number that takes into account the full $z$ evolution [16], including the micromotion. For a finite lattice with edges, the number of topological edge states present in a gap, which is 1 for Fig. 1(c), is directly given by the winding number. Such nontrivial lattices, coined as anomalous Floquet topological insulators [16-19], are unique to periodic driving.

\section{SOLITONLIKE EDGE STATES}

In this work, we seek nonlinear traveling edge states that are highly localized on the zigzag edge- a micrograph of the input facet of the lattice is shown in Fig. 1(d). We consider the evolution governed by Eq. (1) with a single-site input state on the edge. The evolution of the normalized optical intensity along the edge is presented in Figs. 2(a)-2(c) for $\delta=0.18$ and three different renormalized powers $\left(\mathcal{P} \equiv \sum_{s}\left|\Phi_{s}\right|^{2}\right)$ indicated in each figure. For $\mathcal{P} \rightarrow 0$, i.e., in the linear regime, the single-site input state has a large overlap (approximately $68 \%$ ) with the topological edge states; see Fig. 2(a) and Supplemental Movie 1 in [60]. Note that this localized input state (which is, therefore, broad in $k$ space) diffracts along the edge as a result of the curvature of the edge band, shown in Fig. 1(c). This diffraction of the input state can be almost
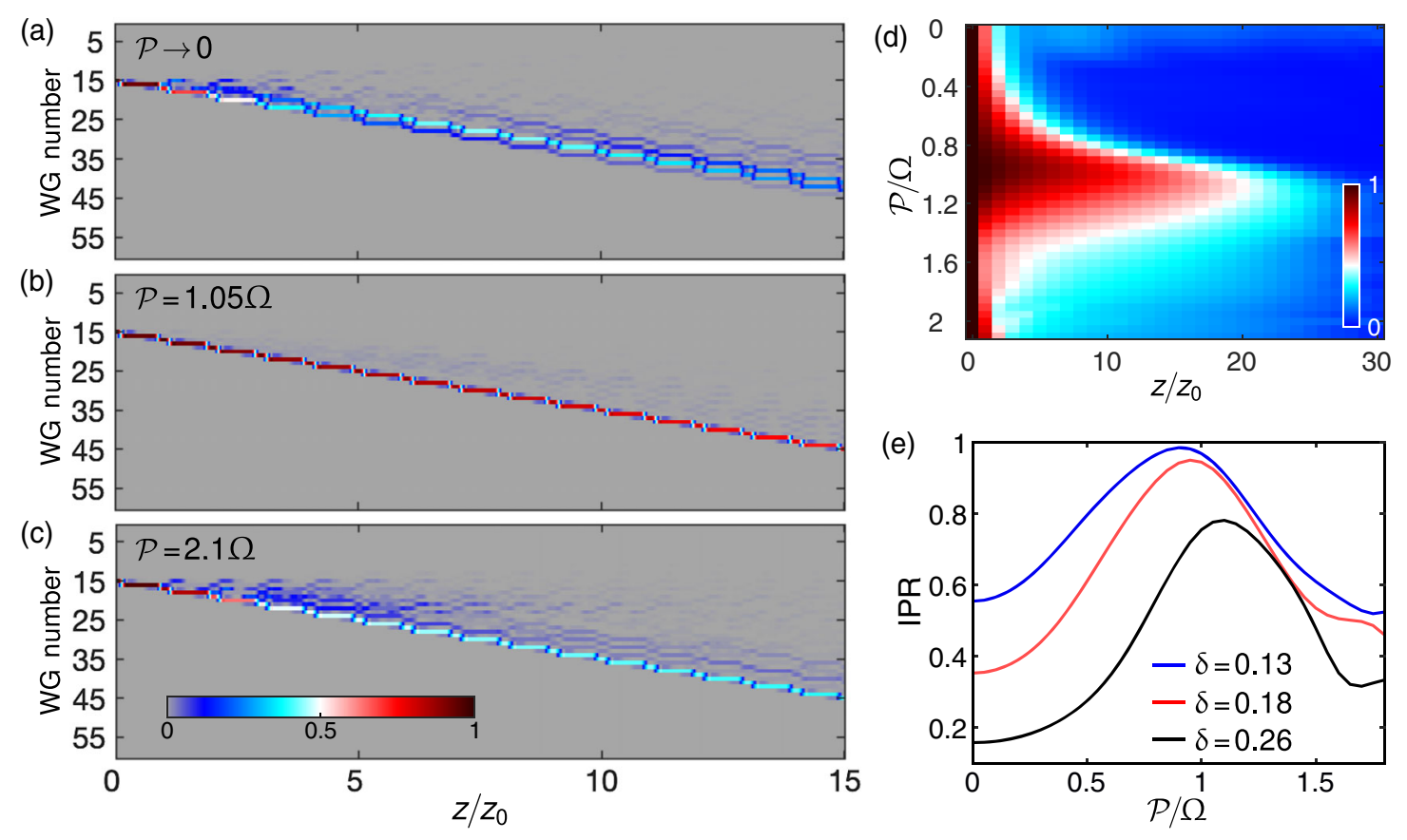

FIG. 2. Unidirectional traveling solitonlike edge states. (a)-(c) Propagation of a single-site input wave packet on the edge of the periodically driven lattice $(\delta=0.18)$ with three different renormalized powers $\mathcal{P}$ indicated in each image. The vertical axis shows the waveguide number along the zigzag edge. For $\mathcal{P} \rightarrow 0$ (the linear regime) and $\mathcal{P}=2.1 \Omega$ [i.e., (a) and (c)], the input state spreads out along the edge as well as penetrates the bulk (not shown here). At a certain intermediate power value (determined by $\delta$ ), the input state propagates unidirectionally with minimal radiation along the edge and into the bulk (b) up to a long propagation distance; see Supplemental Movies 1-3 in [60]. (d) Inverse participation ratio (IPR), calculated after each driving period $z_{0}=2 \pi / \Omega$ as a function of power and propagation distance. A clear peak in the IPR is observed as a function of $\mathcal{P}$, corresponding to the quasilocalized solitonlike state. (e) Variation of IPR at $z=2 z_{0}$ for three different values of $\delta$ that are realized experimentally; see Fig. 3. The IPR at the peak is higher for lower $\delta$, and the value of $\mathcal{P}$ for which the peak occurs increases with $\delta$. 
balanced by nonlinearity, i.e., by increasing the optical power. At a certain value of renormalized power (which is an increasing function of $\delta$ ), the single-site input state propagates unidirectionally with minimal spreading, one lattice constant per driving period, up to a long propagation distance; see Fig. 2(b) and Supplemental Movie 2 in [60]. On the other hand, at higher power, the input state again exhibits a large amount of spreading, as shown in Fig. 2(c) and Supplemental Movie 3 in [60]. We note that the behavior shown in Fig. 2(b) does not occur when only the edge waveguides are present (i.e., the bulk is removed).

The localization of the state as a function of renormalized power and propagation distance can be quantified by the inverse participation ratio, defined as IPR $=\sum_{s}\left|\Phi_{s}\right|^{4} /$ $\left(\sum_{s}\left|\Phi_{s}\right|^{2}\right)^{2}$. When all the light is localized at a single site, the inverse participation ratio is at its maximum value of 1 . Figure 2(d) presents the IPR that is calculated stroboscopically, i.e., after each driving period for the above-mentioned single-site input state. Up to approximately 20 driving cycles, the IPR exhibits a clear peak as a function of renormalized power at a given propagation distance-this peak corresponds to solitonlike wave packet propagation with maximal lifetime. Unlike the intensity distribution along the edge in Figs. 2(a)-2(c), the IPR in Fig. 2(d) accounts for the entire wave function, including the bulk and edge. We note that the localization feature shown in Figs. 2(a)-2(d) can be controlled by tuning $\delta$, i.e., by changing the bulk bandwidth. Indeed, the lifetime of the solitonlike edge state shown in Fig. 2(b) can be increased arbitrarily by reducing $\delta$ (see Appendix B) and diverges as $\delta \rightarrow 0$, i.e., the flat-band limit. To show how this localization peak depends on $\delta$, we compute the IPR as a function of $\mathcal{P}$ for three different values of $\delta$, namely, $0.13,0.18$, and 0.26 , associated with experimentally achieved parameters. Figure 2(e) shows the variation of IPR as a function of $\mathcal{P}$ for each value of $\delta$, calculated after a fixed propagation distance $z=2 z_{0}$. Here, a clear peak in IPR can be observed for all three values of $\delta$.

Two key observations can be made about the peaks in Fig. 2(e): First, the IPR at the peak decreases with $\delta$, and, second, the value of $\mathcal{P}$ at which the peak occurs increases with $\delta$. These features can be explained as follows. In the limit of $\delta \rightarrow 0$ (i.e., flat-band limit), the edge dispersion is linear [53], and the single-site input state overlaps only with the edge states; hence, this input state propagates along the edge without diffraction in the linear regime $(\mathcal{P} \rightarrow 0)$. As $\delta$ increases, there is less of an initial overlap on the edge modes, causing the degree of overall localization to decrease as light spreads into the bulk. Furthermore, the bulk bandwidth increases as $\delta$ increases, implying a faster radiation rate away from the edge and a lower height of the IPR peak. As power increases (at fixed $\delta$ ), coupling to bulk modes is induced at first, and then, as power is further increased, it acts to trap the wave packet on the edge, leading to an increase in IPR with power. At a specific value of the renormalized power $(\mathcal{P}=1.05 \Omega$ for $\delta=0.18)$, the radiation into the bulk is minimal (i.e., the coupling to the bulk modes is reduced [54]), and the state propagates a long distance without significant diffraction along the edge, corresponding to the long-lived solitonlike object and, hence, a peak in the IPR. In other words, for a given set of parameters of the Hamiltonian, the optimal solitonlike wave packet with the longest lifetime is observed at the renormalized power where the IPR exhibits a peak. For further details on nonlinear wave packet dynamics near the peak, see Appendix B. At powers past the IPR peak, there is more power than that which is necessary to form the abovementioned optimal nonlinear quasilocalized state (unlike traditional solitons [34-36]), leading to excess radiation along the edge and into the bulk and a corresponding decrease in IPR. The amount of power required to optimally trap the wave packet along the edge increases with $\delta$, causing a shift in the peak to a higher power with increasing $\delta$. A detailed explanation of the mechanism of localization can be found in Appendix B.

\section{EXPERIMENTS}

We experimentally demonstrate the unidirectional solitonlike edge states by injecting intense laser light into laser-written modulated waveguide arrays with previously mentioned driving protocols. Here, we observe the output states after a fixed propagation distance, as a function of power and for three separate values of $\delta=\{0.13,0.18$, $0.26\}$. Using femtosecond-laser writing [55], three sets of periodically modulated square lattices, each consisting of 56 waveguides and with two driving periods, are fabricated inside a 76-mm-long borosilicate glass substrate. Each waveguide in the lattices supports only the fundamental mode at the operating optical wavelength of $1030 \mathrm{~nm}$. At $z=0$, the lattice sites are well separated ( $27 \mu \mathrm{m}$ intersite spacing) such that all evanescent couplings are negligibly small. To switch on the coupling between any two neighboring waveguides, the interwaveguide separation is reduced to $14 \mu \mathrm{m}$, and then the waveguides are kept straight and parallel where the evanescent coupling reaches a fixed and maximal value; see Figs. 1(b) and 5(a). The couplings are switched off by then separating the waveguides. We control $\delta$ simply by increasing (decreasing) the length of the straight sections of the coupled waveguide pairs in the odd (even) driving step.

Nonlinear characterization of the photonic lattices is performed using intense laser pulses for which the optical field $\Phi_{s}$ is a function of both propagation distance and time. The laser pulses may undergo undesired effects such as self-phase modulation (generating new wavelengths) and chromatic dispersion. To access the self-focusing nonlinearity with a minimal self-phase modulation, we use temporally stretched down-chirped laser pulses. The effect of chromatic dispersion is estimated to be insignificant for the maximum length scale (i.e., $76 \mathrm{~mm}$ propagation distance) considered here. Additionally, nonlinear loss due 

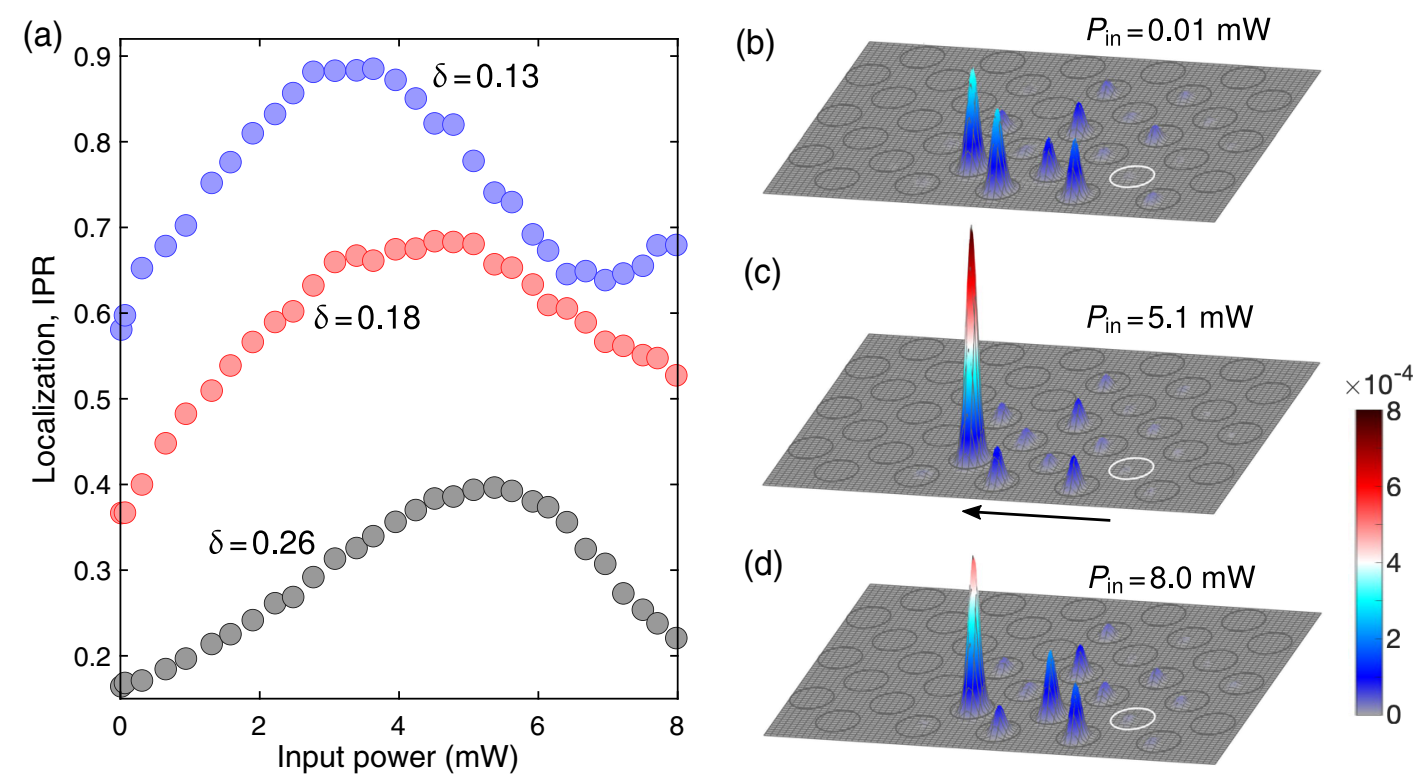

FIG. 3. Observation of solitonlike states traveling unidirectionally along the edge of the topological lattice. (a) Experimentally measured inverse participation ratio at $z=2 z_{0}$ for $\delta=\{0.13,0.18,0.26\}$ - for all cases, a clear peak in the IPR is visible. (b)-(d) Output intensity distributions for $\delta=0.26$ measured at three different power values indicated in each image. The input state propagates two unit cells along the bottom edge with minimal spreading when the average input power is $P_{\text {in }}=5.1 \mathrm{~mW}$, i.e., (c). The white circle in each image indicates the lattice site where light is launched at the input. Each image is normalized, and the field of view is smaller than the lattice size.

to multiphoton absorption is measured to be negligible; see Appendix C.

To experimentally probe the unidirectionally traveling solitonlike edge states, we launch 2 ps laser pulses into the desired edge waveguide [see Fig. 1(d)] and then calculate the inverse participation ratio from the measured intensity profile at the output of the lattices. The relationship between the average input power $P_{\text {in }}$ and the renormalized power $\mathcal{P}$ is experimentally determined to be $\mathcal{P}=0.046 \mathrm{~mm}^{-1}$ per unit $P_{\text {in }}$ in milliwatts; see Appendix C. The nonlinear characterization of the modulated photonic lattices is presented in Fig. 3(a), where the measured IPR at $z=2 z_{0}$ is plotted as a function of the average input power $P_{\text {in }}$ for three different values of $\delta$. As a function of $P_{\text {in }}$, the IPR first increases, exhibits a peak at a particular power, and then decreases for higher power -in all three cases, a clear peak in the IPR is observed, as expected; see Fig. 2(e). We show the measured normalized output intensity patterns $\left|\Phi_{s}\right|^{2} / \mathcal{P}$ for $\delta=0.26$ in Figs. 3(b)-3(d); see also Supplemental Movie 4 in [60]. Figure 3(b) corresponds to the linear case - most of the light propagates unidirectionally (i.e., leftward) along the edge; however, a small amount of light penetrates into the bulk. Importantly, note that the light diffracts, i.e., spreads out, along the edge, which is expected from our numerical results. The solitonlike state, corresponding to the IPR peak, is shown in Fig. 3(c). Figure 3(d) shows the delocalized output intensity at a higher power.

Comparing Figs. 2(e) and 3(a), we observe that the measured IPRs in the linear regime agree well with the expected values; however, the measured heights of the IPR peaks are lower than the expected values, and this effect is more prominent for larger values of $\delta$. This lower IPR is caused by both linear losses and a small background due to

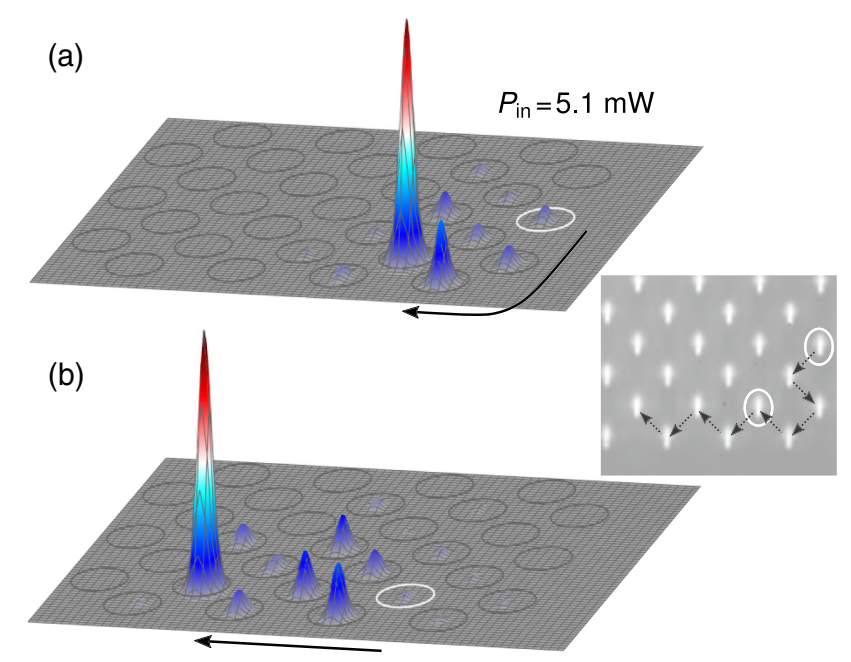

FIG. 4. Solitonlike edge states traveling unidirectionally around a corner. (a),(b) Experimentally measured output intensity patterns for $P_{\text {in }}=5.1 \mathrm{~mW}$ associated with the IPR peak in Fig. 3(a). Here, $\delta=0.26$; the white circles indicate the lattice sites where the light is launched at the input. The inset shows the path of the most intense site. In both cases [i.e., (a) and (b)], the state propagates two lattice constants: the first around the corner, the second along the edge. 
the linear diffraction of the pulse tails (front and rear) in time. Having said that, the observed peaks along with the intensity pattern in Fig. 3(c) clearly agree with the theoretical predictions in Fig. 2 and demonstrate the solitonlike edge states in the topological lattice.

In another set of experiments, we observe the solitonlike state traveling around a corner. Figures 4(a) and 4(b) show the experimentally measured output intensity patterns after two driving periods for two different input excitations indicated by the white circles. These intensity patterns are associated with the IPR peak in Fig. 3(a), i.e., $\delta=0.26$ and $P_{\text {in }}=5.1 \mathrm{~mW}$. These results imply that the solitonlike state can propagate unidirectionally around a corner (i.e., a defect) without backscattering. However, because of nonlinear mode mixing, a defect can, in general, cause extra radiation-see Appendix D for further details.

\section{CONCLUSION}

In conclusion, we have demonstrated solitonlike edge states propagating unidirectionally along the edge of a discrete nonlinear Floquet topological insulator. These nonlinear topological states appear away from the continuum limit, meaning they are highly localized on the edge, and the power required to form such solitonlike waves is comparatively higher than the traditional low-power, spatially broad nonlinear topological waves [40]. This work represents a key development in the understanding and use of topological protection against disorder in nonlinear devices. Since the theory of topological materials is linear at its core, we expect that a new theoretical framework will be necessary for describing the nonlinear optics of topological structures. This will be particularly important if topological protection is to be applied to nonlinear applications such as optical switching, on-chip frequency combs and supercontinuum generation, photon entanglement generation and manipulation, and others.

\section{ACKNOWLEDGMENTS}

The authors acknowledge useful discussions with Daniel Leykam and Jianke Yang. S. M. and M. C. R. gratefully acknowledge support from the Office of Naval Research under Grant No. N00014-18-1-2595, and M.C. R. acknowledges the Packard Foundation under Fellowship No. 2017-66821.

\section{APPENDIX A: TOPOLOGICAL CHARACTERIZATION OF THE PERIODICALLY DRIVEN LATTICE}

In the following sections, we discuss the topological properties of the lattice, show how the lifetime of the solitonlike edge states can be controlled, and present further experimental details. We also discuss what happens when the solitonlike edge state encounters a single defect on the edge with variable on-site energy.
In this section, we discuss the topological characterization of the noninteracting (i.e., linear) periodically driven bipartite square lattice presented in the main text.

Each Bloch band in a lattice system can be characterized by a standard topological invariant such as the Chern number. The Chern numbers of all bands must add up to zero: $\sum_{j} C_{j}=0$. For static systems, the sum of Chern numbers of all bands below a specific band gap is equal to the number of right-moving edge states minus the number of left-moving ones on a given edge. In other words, by computing the band structure considering a strip geometry, the Chern number of any band can be determined by counting the number of topological edge states within the band gaps. In general, this static bulk-edge correspondence is not necessarily applicable to a periodically driven system, as we discuss below.

When a Hamiltonian is driven periodically in time (or $z$ in our photonic system), Floquet theory can be used to analyze the system-quasienergy bands can be computed, and, analogously to static systems, a Chern number can be defined for each quasienergy band. In fact, applying a time-periodic driving, also known as Floquet engineering, has recently emerged as a powerful technique to generate novel effective Hamiltonians and topological phases, which are otherwise inaccessible in static systems.

It was theoretically proposed and then experimentally demonstrated [16-18] that chiral edge states with topological robustness can exist in a Floquet topological insulator with Chern-zero bulk bands, unlike in static systems. The topology of such nontrivial materials, coined as anomalous Floquet topological insulators, is captured by an integer-valued invariant known as the winding number. In this section, we describe a method for the calculation of this topological invariant [16,56] which describes the topology of our two-dimensional lattice system that is periodically driven along the propagation direction $z$ with a driving period $z_{0}=2 \pi / \Omega$.

As mentioned in the main text, the linear tight-binding Hamiltonian associated with our bipartite square lattice (with $\delta$ as the degree of dimerization) changes periodically in $z$ : $H\left(z+z_{0}\right)=H(z)$. The complete modulation cycle is divided into four steps [Fig. 1(b)], and the variation of the couplings $J_{1-4}$ as a function of the propagation distance is shown in Fig. 5(a). For the $m$ th driving step, we define $\Lambda_{m} \equiv$ $\int d z J_{m}(z)$ (the integration is taken over each driving step). Note that the steplike coupling functions have a fixed gradual rise and fall time; however, the flat section is dimerized such that $\Lambda_{1,3}=\pi / 2(1+\delta)$ and $\Lambda_{2,4}==\pi / 2(1-\delta)$.

The quasienergy spectrum of this $z$-periodic lattice is obtained by diagonalizing the propagator over one complete period, where the propagator is given by $\hat{U}\left(z_{0}\right)=\mathcal{T} \exp \left[-i \int_{0}^{z_{0}} d \tilde{z} \hat{H}(\tilde{z})\right]$, where $\mathcal{T}$ indicates the time ordering in $\tilde{z}$. We numerically calculate the quasienergy spectrum as a function of $\delta$ [Fig. 5(b)]. For all values of $\delta$ 
(a)

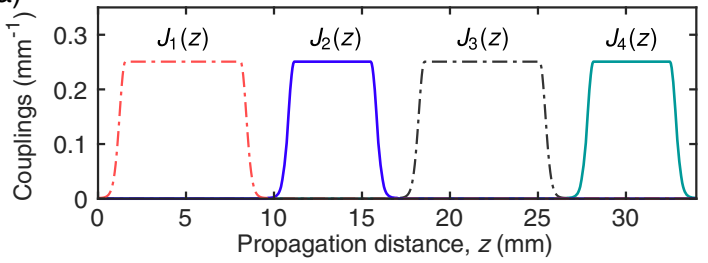

(b)

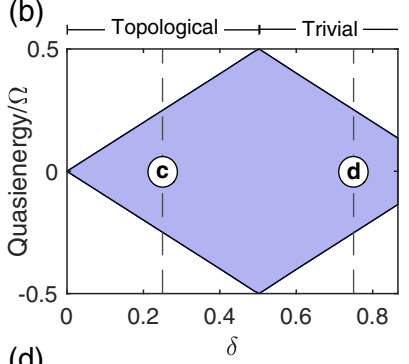

(d)

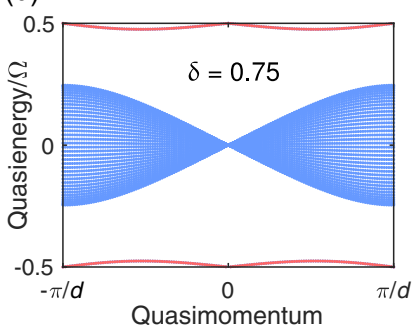

(c)

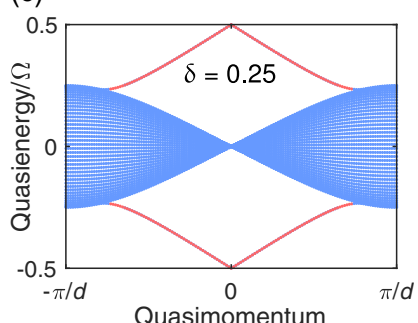

(e)

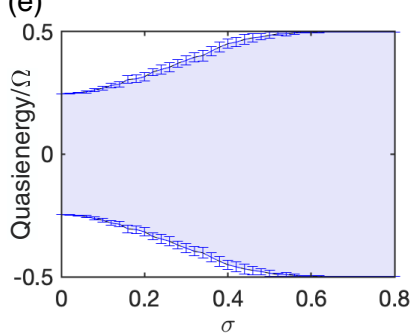

FIG. 5. (a) The variation of the couplings $J_{1-4}$ as a function of the propagation distance. The couplings resemble a steplike function with a fixed gradual rise and fall time. (b) Bulk quasienergy spectrum of the driven bipartite lattice as a function of $\delta$. The blue (white) region indicates the linear bulk band (band gap); the band gap closes at $\delta=0.5$. (c),(d) Quasienergy spectra calculated using a strip geometry for $\delta=0.25$ (topological) and $\delta=0.75$ (trivial), respectively, indicated by the vertical dashed lines in (b). Topological unidirectional edge states (one per edge) appear for $0 \leq \delta<0.5$. For $\delta>0.5$, the edge states are trivial. (e) Quasienergy spectrum as a function of disorder strength $\sigma$ in $\Lambda_{1-4}$. The error bars indicate the standard deviation of maximum (minimum) quasienergies. The unavoidable small disorder present in the experiments $\left(\sigma_{\exp }=0.05\right)$ does not close the band gap.

considered here, there are two ungapped bulk bands and one band gap which closes at $\delta=0.5$.

To capture the existence of edge states, we then calculate the spectrum using a strip geometry terminated in the vertical direction and arrayed periodically along the horizontal direction. Topological unidirectional edge states (one per edge) appear for $0 \leq \delta<0.5$. As an example, we present the spectrum associated with $\delta=0.25$, where the red lines indicate the topological edge states on the upper and lower edges of the lattice [Fig. 5(c)]. For $\delta>0.5$, we observe topologically trivial edge states; see an example in Fig. 5(d). By counting the number of topological edge states on a given edge, we conclude that the Floquet winding number is unity for $0 \leq \delta<0.5$ and zero for $\delta>0.5$.

In our experiments, we observe small but unavoidable disorder in $\Lambda_{1-4}$ with an estimated standard deviation

$\sigma_{\text {exp }}=0.05$. To verify that this small disorder does not close the band gap, we calculate the bulk quasienergy spectrum as a function of the disorder strength $\sigma$ in $\Lambda_{1-4}$; see Fig. 5(e). For these calculations, we redefine $\tilde{\Lambda}_{1-4}=\Lambda_{1-4}(1+\tilde{\lambda})$, where $\tilde{\lambda}$ is a random variation of $\Lambda_{1-4}$ taken from the normal distribution with zero mean and a standard deviation parameter $\sigma$. For each $\sigma$ value, we consider 25 separate realizations of disorder, and the error bars in Fig. 5(e) indicate the standard deviation of the maximum (minimum) quasienergies. It is evident that the quasienergy gap does not close until $\sigma \lesssim 0.4$.

\section{APPENDIX B: FINITE LIFETIME OF SOLITONLIKE EDGE STATES}

Because of the discreteness of the system and the associated Peierls-Nabarro barrier experienced by the wave packet as it moves through the lattice [49], confined traveling nonlinear waves will not, in general, live forever and cannot, therefore, be called solitons. As shown in the main text, the optimal solitonlike wave packet propagates with minimal radiation. In this section, we discuss the dependence of the lifetime of the solitonlike edge wave packets on the dimerization parameter $\delta$. Nonlinear wave packet dynamics near the IPR peak is also elaborated.

In the main text, we present numerical results showing unidirectional traveling solitonlike edge states, in Fig. 2, for $\delta=0.18$. Figure 6(a) shows the same variation of IPR, presented in Fig. 2(d), for a longer propagation distance. Up to approximately 20 driving cycles, the IPR exhibits a clear peak as a function of the renormalized power at a
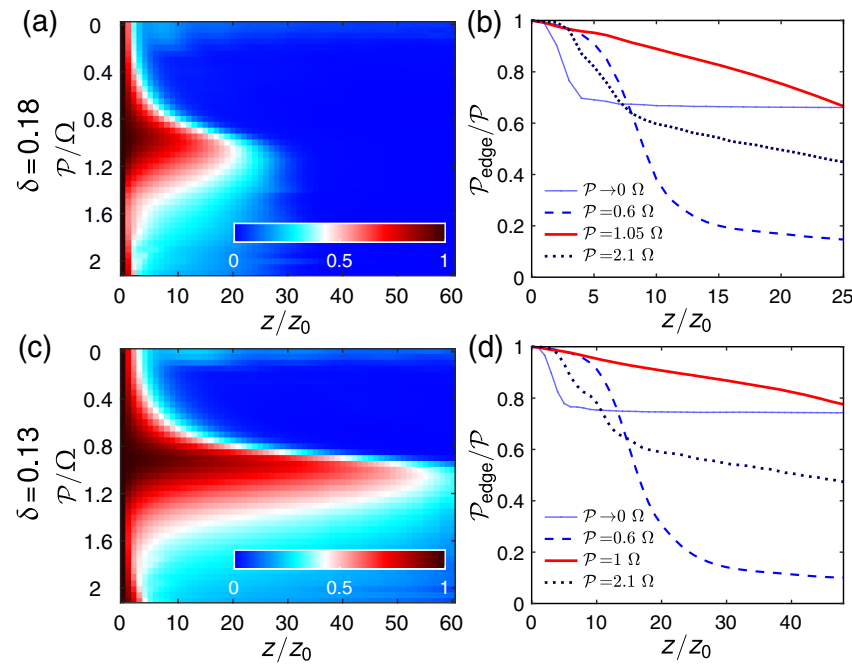

FIG. 6. IPR and power on the edge for two different values of $\delta$. (a),(c) The variation of IPR, calculated after each driving period, as a function of renormalized power and propagation distance for $\delta=0.18$ and 0.13 , respectively. (b),(d) The variation of the fraction of total normalized power on the zigzag edge, for different values of renormalized powers indicated in the figures, corresponding to $\delta=0.18$ and 0.13 , respectively. 
given propagation distance. In this case, the fraction of light on the zigzag edge $\mathcal{P}_{\text {edge }} / \mathcal{P}$ is plotted in Fig. 6(b) for four different renormalized powers $\mathcal{P}$, indicated in the figure. In the linear regime $\mathcal{P} \rightarrow 0$, the single-site input state largely overlaps (approximately 68\%) with the edge states; hence, some light penetrates into the bulk in the beginning $\left(0 \leq z / z_{0} \lesssim 5\right)$, and, then, $\mathcal{P}_{\text {edge }} / \mathcal{P}$ remains unalteredsee the solid blue line in Fig. 6(b). As shown in Supplemental Movie 1 in [60], the remaining light on the edge spreads because of the curvature of the edge band.

When nonlinearity is introduced, the edge overlap $\sum_{i}\left|\left\langle\Phi(z) \mid \psi_{i}\right\rangle\right|^{2}$ [i.e., the overlap of the linear topological edge states $\psi_{i}$ with the normalized wave packet $\left.\Phi(z)\right]$ varies as a function of the propagation distance and the strength of nonlinearity. At small power $\mathcal{P}=0.6 \Omega$, the wave packet strongly couples to bulk modes and, thus, radiates away from the edge; see the blue dashed line in Fig. 6(b). Furthermore, this is below the power threshold for the formation of the optimal solitonlike wave packet, meaning that there is still significant diffraction along the edge. At a specific value of the renormalized power
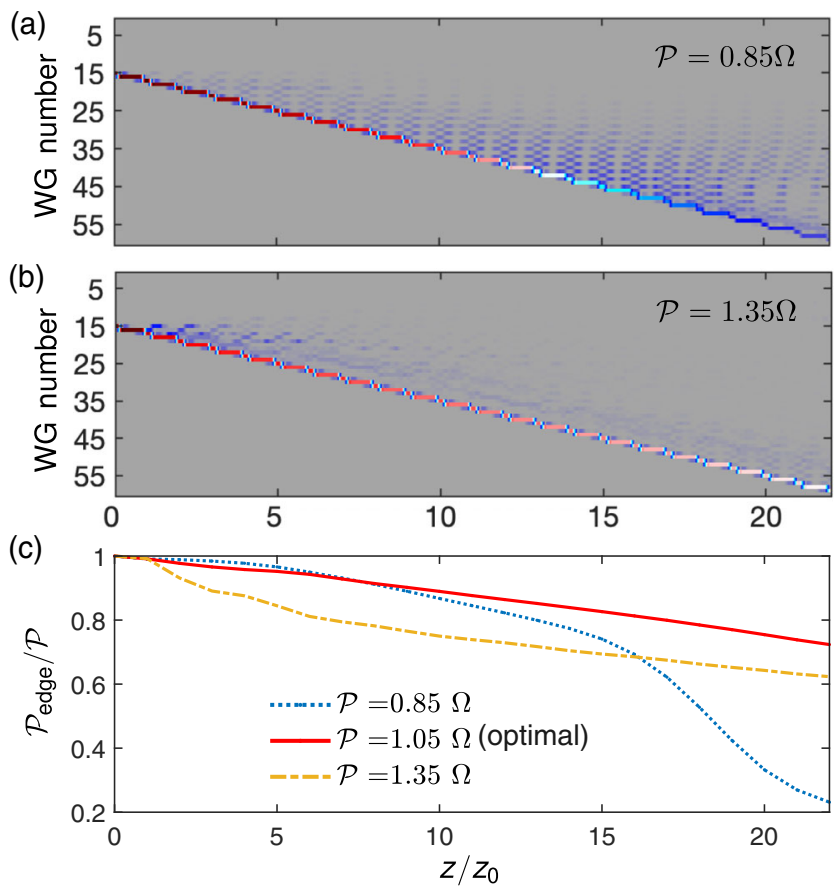

FIG. 7. Nonlinear edge states near the power value associated with the IPR peak. (a),(b) Propagation of a single-site input wave packet on the edge of the periodically driven lattice $(\delta=0.18)$ with two different renormalized powers $\mathcal{P}$ indicated in each plot. The optimal solitonlike propagation of the nonlinear edge state with minimal radiation (i.e., with the longest lifetime) is associated with the IPR peak; see Fig. 2(b) and the solid red line in (c). As shown in (a) and (b), there exist similar nonlinear states with excess radiation near (i.e., before and after) the IPR peak. (c) The variation of the fraction of total normalized power on the zigzag edge, for different values of renormalized powers indicated in the figures.
$\mathcal{P}=1.05 \Omega$ associated with the IPR peak, the radiation into the bulk reduces (i.e., the edge overlap increases), and, importantly, the state propagates a long distance without significant diffraction along the edge, corresponding to the long-lived solitonlike object and, hence, a peak in the IPR [see the solid red line in Fig. 6(b)]. At higher power $\mathcal{P}=2.1 \Omega$, the radiation increases again (see the dotted dark blue line), and the state diffracts during propagation.

In Figs. 7(a) and 7(b), the propagation of nonlinear edge states is shown in the vicinity of the power value associated with the IPR peak. For a given set of parameters, the solitonlike state associated with the IPR peak has minimal radiation and, hence, the longest lifetime; see Figs. 2(b) and 7(c). Near the IPR peak (i.e., at a smaller and/or larger value of renormalized power), the nonlinear edge states propagate with excess radiation; see Fig. 7(c).

The radiation rate of the optimal solitonlike edge state increases with increasing $\delta$. Figures 6(c) and 6(d) present the same results as Figs. 6(a) and 6(b) except with a lower value of $\delta=0.13$. In this case, the clear peak in the IPR can be observed up to a longer propagation distance $z \approx 55 z_{0}$. The result in Fig. 6(d) is qualitatively similar to Fig. 6(b); however, the important difference is that the dynamics is slower at this smaller value of $\delta$-the optimal solitonlike edge state (the solid red line) has a comparatively longer lifetime. In the limit of $\delta \rightarrow 0$, the edge spectrum has linear dispersion [53], and the bulk is dispersionless. In this case, nondiffracting unidirectional edge transport is observed for an arbitrarily long propagation distance in the limit of zero nonlinearity, $\mathcal{P} \rightarrow 0$.

\section{APPENDIX C: MORE EXPERIMENTAL DETAILS}

\section{Fabrication}

Topological photonic lattices consisting of periodically modulated single-mode optical waveguides are created using femtosecond (fs) laser writing, an on-chip device fabrication technique $[55,57,58]$. This technique allows us to precisely control the waveguide paths in a threedimensional geometry, which is crucial for realizing the nontrivial topology considered in this work. Laser pulses at $1030 \pm 4 \mathrm{~nm}$ wavelength, $500 \mathrm{kHz}$ pulse repetition rate, and 260 fs pulse width are generated using a commercially available Yb-doped (Menlo BlueCut) fiber laser system. Each waveguide in the lattice is inscribed by translating a borosilicate (Corning Eagle XG) glass substrate-which is mounted on a high-precision $x-y-z$ (Aerotech) translation stage-once through the focus of the femtosecond laser beam. The laser pulse energy and translation speed of fabrication are optimized to obtain low-loss single-mode optical waveguides near the $1030 \mathrm{~nm}$ wavelength. The maximal insertion (propagation + bend + input coupling) loss for the entire lattice is measured to be $7.5 \mathrm{~dB}$, and no significant polarization-dependent loss is detected in our experiments. 


\section{Nonlinear characterization}

Figure 8(a) presents a simplified schematic of the nonlinear characterization setup. We use laser pulses at $5 \mathrm{kHz}$ repetition rate generated by the Menlo BlueCut system. The average input power can be tuned in our experiments by using a combination of a half wave plate and a polarizing beam splitter. A parallel grating pair is used to temporally stretch (and down-chirp) the pulses to $2 \mathrm{ps}$. To gauge the nonlinear loss due to multiphoton absorption, we measure the average output power as a function of the average input power for all nonlinear characterizations, and a linear variation is observed, as shown in Fig. 8(b). This linear variation of $P_{\text {out }}$ with the input power $P_{\text {in }}$ implies that nonlinear losses can be ignored in our experiments.

As mentioned in the main text, the temporal shape of the laser pulses can cause undesired effects such as self-phase modulation (i.e., generation of new wavelengths) and chromatic dispersion. To access self-focusing nonlinearity with a minimal self-phase modulation, we use temporally stretched and down-chirped laser pulses. In this situation, a maximal spectral width of $<20 \mathrm{~nm}$ is observed in our experiments; see Fig. 8(c). In this wavelength range, the evanescent coupling $J$ varies only by $\Delta J / J= \pm 4 \%$, which is of the order of the unavoidable small disorder present in the lattice. Hence, we can safely ignore the effects of self-phase modulation in our experiments. The effect of chromatic dispersion is negligibly small for the maximal propagation distance $(76 \mathrm{~mm})$ considered in our experiments [44].

\section{Estimation of $\mathcal{P}$}

The renormalized power $\mathcal{P}$ is experimentally calibrated by characterizing a two-waveguide directional coupler with
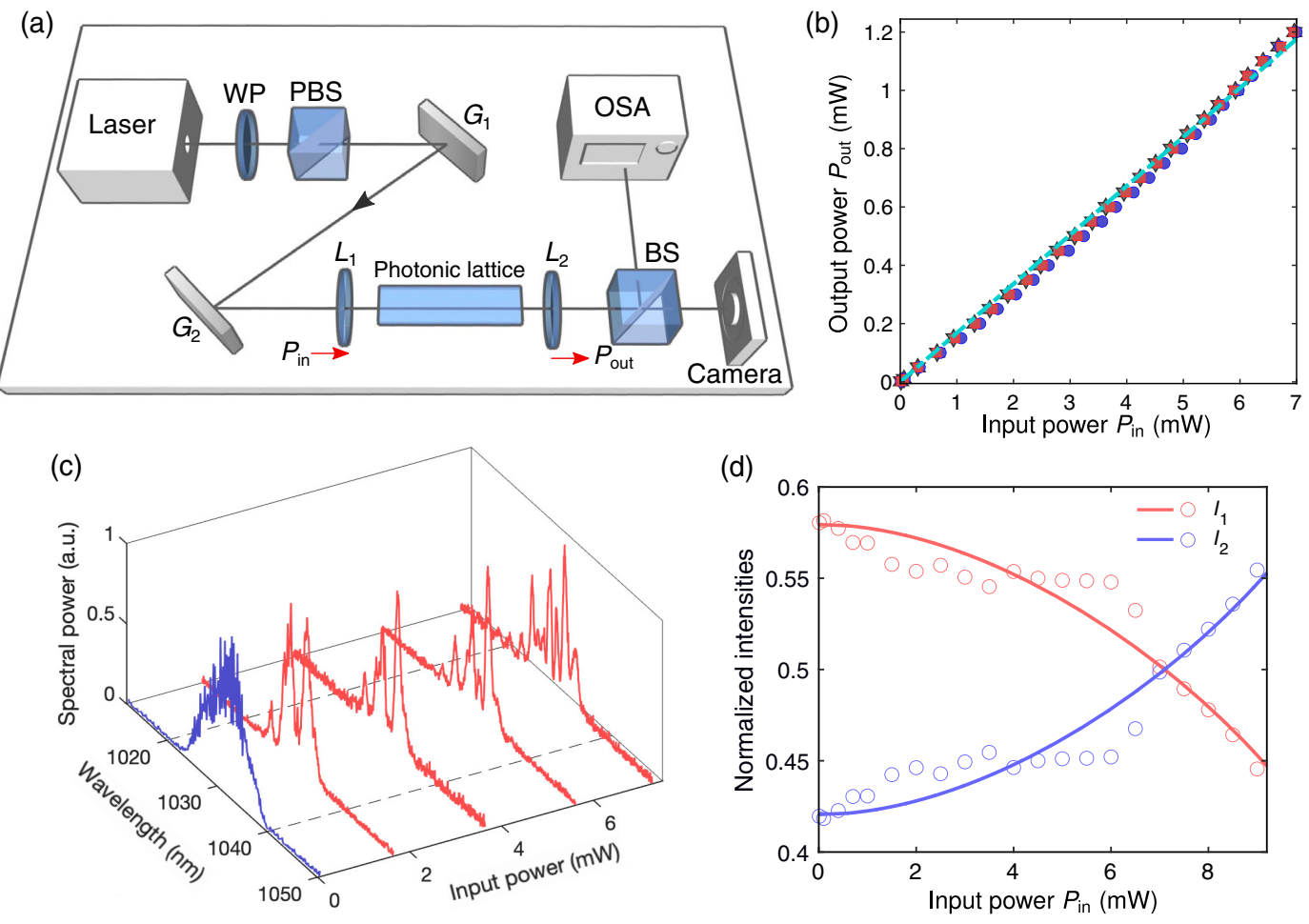

FIG. 8. Nonlinear characterization. (a) Simplified schematic of the nonlinear characterization setup. Here, WP is a half wave plate, PBS is a polarizing beam splitter, $G_{1,2}$ is a parallel grating pair, $L_{1,2}$ are air-coated convex lenses, and BS is a beam splitter. Laser pulse trains at $1030 \pm 4 \mathrm{~nm}$ wavelength, $5 \mathrm{kHz}$ pulse repetition rate, and $260 \mathrm{fs}$ pulse duration are generated using a Yb-doped fiber laser (Menlo BlueCut) system. The parallel grating pair is used to temporally stretch (and down-chirp) the pulses to 2 ps. The intensity patterns at the output of the photonic lattices are imaged on a CMOS camera, and the optical spectrum (intensity vs wavelength) is measured using an optical spectrum analyzer (OSA). (b) The variation of average output power $P_{\text {out }}$ with the input power $P_{\text {in }}$. The linear variation implies that nonlinear losses due to multiphoton absorption processes can be ignored in our experiments. Here, three datasets are shown, and the dashed line indicates a linear fit. (c) Self-phase modulation (SPM) induced by Kerr nonlinearity. Here, the normalized spectral powers are shown for five sets of average input powers. The spectral width in the linear regime (shown in blue) is approximately $8 \mathrm{~nm}$ (FWHM). At higher powers, the spectrum changes due to SPM; however, the maximal spectral width after $76 \mathrm{~mm}$ of propagation is measured to be less than $20 \mathrm{~nm}$ for the maximal required nonlinearity. (d) The renormalized power $\mathcal{P}$ is estimated by characterizing a nonlinear directional coupler. The data in red (blue) are the measured normalized intensity $I_{1(2)}$ at the output of waveguide 1 (2) (light is launched into waveguide 1). The solid lines are obtained by solving Eq. (C1) and fitting $\mathcal{P}$ at the input to be $0.046 \mathrm{~mm}^{-1}$ for unit input power in milliwatts. 
a known coupling strength and linear loss coefficient. The paraxial propagation of light in a nonlinear directional coupler is governed by [59]

$$
i \frac{\partial}{\partial z} \Phi_{1,2}=-J \Phi_{2,1}-\left|\Phi_{1,2}\right|^{2} \Phi_{1,2}-i \alpha \Phi_{1,2}
$$

where $J$ is the coupling strength, $\alpha$ is a measure of linear loss, and $\left|\Phi_{1,2}\right|^{2}$ is proportional to the optical power at waveguide 1 and 2 , respectively. It should be mentioned that $\mathcal{P}=\left(\left|\Phi_{1}\right|^{2}+\left|\Phi_{2}\right|^{2}\right)$ is not a conserved quantity during propagation when optical losses are present-here, we experimentally estimate $\mathcal{P}(z=0)$. In the experiments, we launch light at waveguide 1 ; the variation of normalized output intensities $\left|\Phi_{1,2}\right|^{2} /\left(\left|\Phi_{1}\right|^{2}+\left|\Phi_{2}\right|^{2}\right)$ with average input power is presented in Fig. 8(d). The solid lines are obtained by solving Eq. (C1) and fitting $\mathcal{P}$ at the input to be $0.046 \mathrm{~mm}^{-1}$ for unit input power in milliwatts.

\section{APPENDIX D: SOLITONLIKE EDGE STATE ENCOUNTERING A SINGLE DEFECT ON THE EDGE OF THE TOPOLOGICAL LATTICE}

In the main text, we experimentally demonstrate that the solitonlike edge state can travel around a corner without backscattering. In this section, we consider a more general situation, i.e., what happens when the solitonlike edge state encounters a single defect on the edge with variable on-site energy. We show that the solitonlike nonlinear state is not largely affected by a weak defect; however, it can scatter and fall apart in the presence of a strong one. Importantly, because of the underlying topological properties, backscattering is not allowed even in the nonlinear case, simply because of the absence of a backward-propagating linear mode.

Consider the periodically driven square lattice discussed in the main text with $\delta=0.18$. As shown in Fig. 9(a), the on-site energy of a single site on the edge is detuned by $\Delta$ with respect to the other waveguides. This corresponds to adding an extra term $-\Delta \delta_{s, s_{0}} \Phi_{s}$ on the right-hand side of
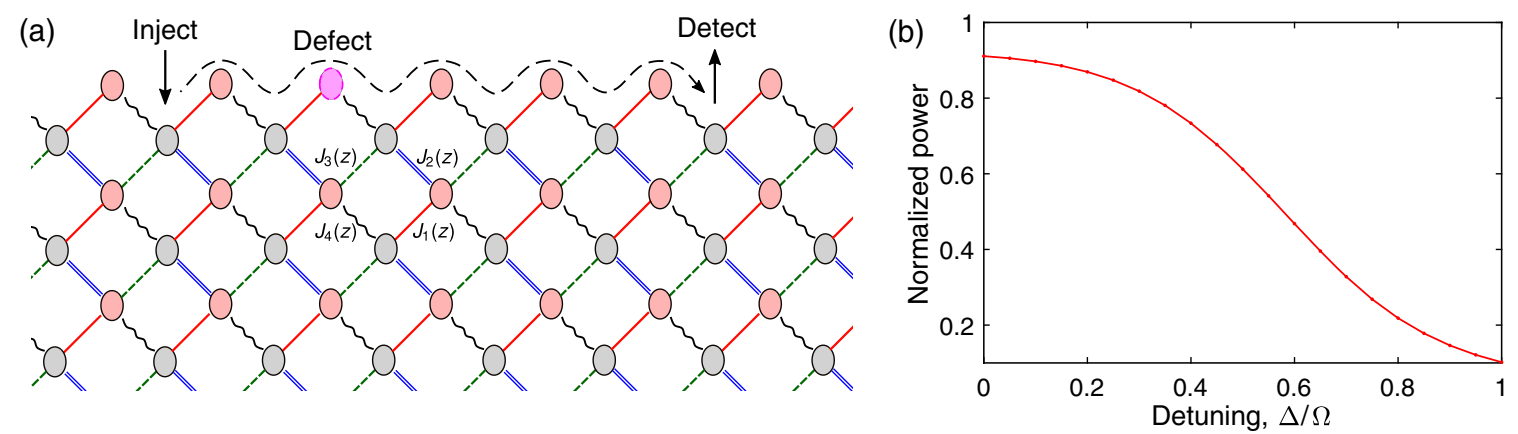

(c)

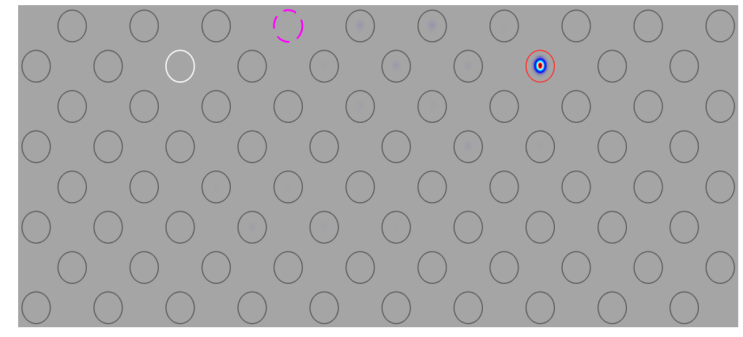

(e)

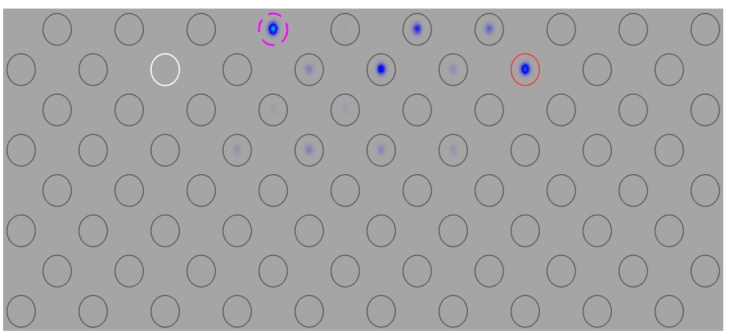

(d)

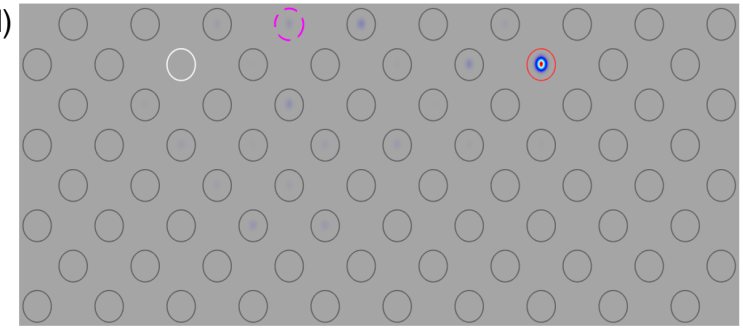

(f)

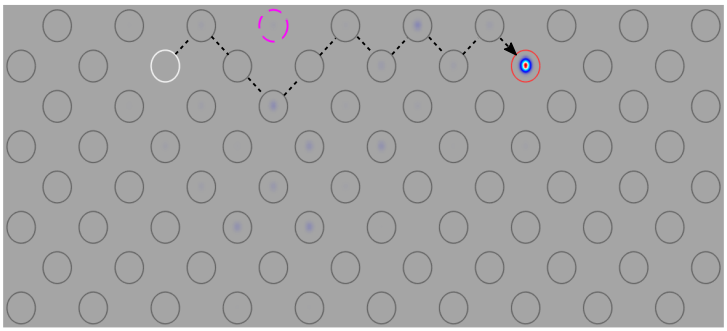

FIG. 9. A single defect on the edge. (a) Schematic of the driven square lattice discussed in the main text with $\delta=0.18$ and a detuned site indicated by the magenta color. The nonlinear solitonlike state is injected at a single site (indicated by an arrow) and then detected after the state travels through a defect site. (b) Normalized peak power detected after propagation of $z=5 z_{0}$ as a function of the detuning of the on-site energy of the defect site. (c)-(e) Output intensity pattern associated with (b) for three different detunings $\Delta / \Omega=0,0.3$, and 0.8 , respectively. For zero or small disorder [i.e., (c) and (d), respectively], the solitonlike state propagates unidirectionally with minimal radiation. For a comparatively larger disorder $\Delta / \Omega=0.8$, the solitonlike state falls apart as shown in (e). (f) For an extensive detuning $\Delta / \Omega=20$, the nonlinear state travels around the defect site- this is similar to the situation wherein the defect site is removed. The white circle indicates the site at which light is injected at the input. 
Eq. (1), where $s_{0}$ indexes the detuned site and $\delta_{i, j}$ is the Kronecker delta function. In our numerical calculations, the optimal solitonlike state is injected at a single site [indicated by an arrow in Fig. 9(a)] and then detected after the state travels through the detuned site. The normalized peak power after propagation of $z=5 z_{0}$ is plotted in Fig. 9(b) as a function of the detuned on-site energy of the defect site. The associated output intensity patterns for three different detunings, $\Delta / \Omega=0,0.3$, and 0.8 , are shown in Figs. 9(c)-9(e), respectively. For zero or a small detuning [i.e., Figs. 9(c) and A5(d), respectively], the solitonlike state propagates unidirectionally with small radiation. Unlike the linear case, a small on-site defect can increase the radiation and, hence, reduce the lifetime of the nonlinear state. For a comparatively larger detuning $\Delta / \Omega=0.8$, which is of the order of the band gap, the solitonlike state falls apart, as shown in Fig. 9(e). In Fig. 9(f), we consider $\Delta / \Omega=20$, implying that the defect site is effectively isolated from the lattice. In this case, the nonlinear state travels around the defect site.

In summary, the solitonlike state in our discrete photonic lattice can travel unidirectionally without backscattering, because there is no state available to backscatter. Under certain conditions, the nonlinearity can enhance radiation because of the complex nonlinear mode mixing.

\section{APPENDIX E: DESCRIPTION OF THE SUPPLEMENTAL MOVIES}

\section{Movies 1-3}

Propagation of a single-site input wave packet on the edge of the periodically driven lattice $(\delta=0.18)$ with three different renormalized powers $\mathcal{P}$, indicated in each movie. The white circle in each image indicates the site where the light is launched at the input. Unlike Figs. 2(a)-2(c) in the main text, the full two-dimensional lattice is shown in these movies. For $\mathcal{P} \rightarrow 0$ (Movie 1) and $\mathcal{P}=2.1 \Omega$ (Movie 3), the input state spreads out along the edge as well as penetrates the bulk. At a certain intermediate power value $(\mathcal{P}=1.05 \Omega$, Movie 2$)$, the input state propagates unidirectionally up to a long propagation distance with minimal radiation.

\section{Movie 4}

Experimentally measured intensity patterns (left) and calculated inverse participation ratio (right) at propagation distance $z=2 z_{0}$ for the driving parameter $\delta=0.26$-a clear peak in the IPR is visible as a function of input power. The input state propagates two unit cells along the bottom edge with minimal spreading when the average input power is near $P_{\text {in }}=5.1 \mathrm{~mW}$. The white circle indicates the lattice site where light is launched at the input. Each image is normalized, and the field of view is smaller than the lattice size.
[1] S. Raghu and F. D. M. Haldane, Analogs of Quantum-HallEffect Edge States in Photonic Crystals, Phys. Rev. A 78, 033834 (2008).

[2] Z. Wang, Y. Chong, J. D. Joannopoulos, and M. Soljačić, Observation of Unidirectional Backscattering-Immune Topological Electromagnetic States, Nature (London) 461, 772 (2009).

[3] M. C. Rechtsman, J. M. Zeuner, Y. Plotnik, Y. Lumer, D. Podolsky, F. Dreisow, S. Nolte, M. Segev, and A. Szameit, Photonic Floquet Topological Insulators, Nature (London) 496, 196 (2013).

[4] M. Hafezi, S. Mittal, J. Fan, A. Migdall, and J. Taylor, Imaging Topological Edge States in Silicon Photonics, Nat. Photonics 7, 1001 (2013).

[5] T. Ozawa, H. M. Price, A. Amo, N. Goldman, M. Hafezi, L. Lu, M. C. Rechtsman, D. Schuster, J. Simon, O. Zilberberg, and I. Carusotto, Topological Photonics, Rev. Mod. Phys. 91, 015006 (2019).

[6] M. Hafezi, E. A. Demler, M. D. Lukin, and J. M. Taylor, Robust Optical Delay Lines with Topological Protection, Nat. Phys. 7, 907 (2011).

[7] G. Harari, M. A. Bandres, Y. Lumer, M. C. Rechtsman, Y. D. Chong, M. Khajavikhan, D. N. Christodoulides, and M. Segev, Topological Insulator Laser: Theory, Science 359, eaar4003 (2018).

[8] M. A. Bandres, S. Wittek, G. Harari, M. Parto, J. Ren, M. Segev, D. N. Christodoulides, and M. Khajavikhan, Topological Insulator Laser: Experiments, Science 359, eaar4005 (2018).

[9] J. Guglielmon and M. C. Rechtsman, Broadband Topological Slow Light through Higher Momentum-Space Winding, Phys. Rev. Lett. 122, 153904 (2019).

[10] K. v. Klitzing, G. Dorda, and M. Pepper, New Method for High-Accuracy Determination of the Fine-Structure Constant Based on Quantized Hall Resistance, Phys. Rev. Lett. 45, 494 (1980).

[11] D. J. Thouless, M. Kohmoto, M. P. Nightingale, and M. den Nijs, Quantized Hall Conductance in a Two-Dimensional Periodic Potential, Phys. Rev. Lett. 49, 405 (1982).

[12] B. I. Halperin, Quantized Hall Conductance, CurrentCarrying Edge States, and the Existence of Extended States in a Two-Dimensional Disordered Potential, Phys. Rev. B 25, 2185 (1982).

[13] F. D. M. Haldane, Model for a Quantum Hall Effect without Landau Levels: Condensed-Matter Realization of the "Parity Anomaly", Phys. Rev. Lett. 61, 2015 (1988).

[14] C. L. Kane and E. J. Mele, Quantum Spin Hall Effect in Graphene, Phys. Rev. Lett. 95, 226801 (2005).

[15] B. A. Bernevig, T. L. Hughes, and S.-C. Zhang, Quantum Spin Hall Effect and Topological Phase Transition in HgTe Quantum Wells, Science 314, 1757 (2006).

[16] M. S. Rudner, N. H. Lindner, E. Berg, and M. Levin, Anomalous Edge States and the Bulk-Edge Correspondence for Periodically Driven Two-Dimensional Systems, Phys. Rev. X 3, 031005 (2013).

[17] S. Mukherjee, A. Spracklen, M. Valiente, E. Andersson, P. Öhberg, N. Goldman, and R. R. Thomson, Experimental Observation of Anomalous Topological Edge Modes in a Slowly Driven Photonic Lattice, Nat. Commun. 8, 13918 (2017). 
[18] L. J. Maczewsky, J. M. Zeuner, S. Nolte, and A. Szameit, Observation of Photonic Anomalous Floquet Topological Insulators, Nat. Commun. 8, 13756 (2017).

[19] S. Mukherjee, H. K. Chandrasekharan, P. Öhberg, N. Goldman, and R. R. Thomson, State-Recycling and TimeResolved Imaging in Topological Photonic Lattices, Nat. Commun. 9, 4209 (2018).

[20] G. Jotzu, M. Messer, R. Desbuquois, M. Lebrat, T. Uehlinger, D. Greif, and T. Esslinger, Experimental Realization of the Topological Haldane Model with Ultracold Fermions, Nature (London) 515, 237 (2014).

[21] K. Wintersperger, C. Braun, F. N. Ünal, A. Eckardt, M. Di Liberto, N. Goldman, I. Bloch, and M. Aidelsburger, Realization of Anomalous Floquet Topological Phases with Ultracold Atoms, Nat. Phys. 16, 1058 (2020).

[22] L. M. Nash, D. Kleckner, A. Read, V. Vitelli, A. M. Turner, and W. T. Irvine, Topological Mechanics of Gyroscopic Metamaterials, Proc. Natl. Acad. Sci. U.S.A. 112, 14495 (2015).

[23] R. Süsstrunk and S. D. Huber, Observation of Phononic Helical Edge States in a Mechanical Topological Insulator, Science 349, 47 (2015).

[24] J. Ningyuan, C. Owens, A. Sommer, D. Schuster, and J. Simon, Time- and Site-Resolved Dynamics in a Topological Circuit, Phys. Rev. X 5, 021031 (2015).

[25] T. Karzig, C.-E. Bardyn, N. H. Lindner, and G. Refael, Topological Polaritons, Phys. Rev. X 5, 031001 (2015).

[26] A. V. Nalitov, D. D. Solnyshkov, and G. Malpuech, Polariton z Topological Insulator, Phys. Rev. Lett. 114, 116401 (2015).

[27] S. Klembt, T. H. Harder, O. A. Egorov, K. Winkler, R. Ge, M. A. Bandres, M. Emmerling, L. Worschech, T. C. H. Liew, M. Segev et al., Exciton-Polariton Topological Insulator, Nature (London) 562, 552 (2018).

[28] P. Delplace, J. B. Marston, and A. Venaille, Topological Origin of Equatorial Waves, Science 358, 1075 (2017).

[29] A. Shabat and V. Zakharov, Exact Theory of TwoDimensional Self-Focusing and One-Dimensional SelfModulation of Waves in Nonlinear Media, Sov. Phys. JETP 34, 62 (1972), http://zakharov75.itp.ac.ru/static/local/zve75/ zakharov/1972/1972-05-e_034_01_0062.pdf.

[30] M. J. Ablowitz and H. Segur, Solitons and the Inverse Scattering Transform (Society for Industrial and Applied Mathematics, Philadelphia, 1981).

[31] R. Camassa and D. D. Holm, An Integrable Shallow Water Equation with Peaked Solitons, Phys. Rev. Lett. 71, 1661 (1993).

[32] A. Barthelemy, S. Maneuf, and C. Froehly, Propagation Soliton et Auto-confinement de Faisceaux Laser par Non linearité Optique de Kerr, Opt. Commun. 55, 201 (1985).

[33] D. N. Christodoulides and R. I. Joseph, Discrete SelfFocusing in Nonlinear Arrays of Coupled Waveguides, Opt. Lett. 13, 794 (1988).

[34] M. Segev, B. Crosignani, A. Yariv, and B. Fischer, Spatial Solitons in Photorefractive Media, Phys. Rev. Lett. 68, 923 (1992).

[35] H. S. Eisenberg, Y. Silberberg, R. Morandotti, A. R. Boyd, and J.S. Aitchison, Discrete Spatial Optical Solitons in Waveguide Arrays, Phys. Rev. Lett. 81, 3383 (1998).
[36] J. W. Fleischer, M. Segev, N. K. Efremidis, and D. N. Christodoulides, Observation of Two-Dimensional Discrete Solitons in Optically Induced Nonlinear Photonic Lattices, Nature (London) 422, 147 (2003).

[37] J. Denschlag, J. E. Simsarian, D. L. Feder, C. W. Clark, L. A. Collins, J. Cubizolles, L. Deng, E. W. Hagley, K. Helmerson, W. P. Reinhardt et al., Generating Solitons by Phase Engineering of a Bose-Einstein Condensate, Science 287, 97 (2000).

[38] S. Burger, K. Bongs, S. Dettmer, W. Ertmer, K. Sengstock, A. Sanpera, G. V. Shlyapnikov, and M. Lewenstein, Dark Solitons in Bose-Einstein Condensates, Phys. Rev. Lett. 83, 5198 (1999).

[39] Y. Lumer, Y. Plotnik, M. C. Rechtsman, and M. Segev, SelfLocalized States in Photonic Topological Insulators, Phys. Rev. Lett. 111, 243905 (2013).

[40] M. J. Ablowitz, C. W. Curtis, and Y.-P. Ma, Linear and Nonlinear Traveling Edge Waves in Optical Honeycomb Lattices, Phys. Rev. A 90, 023813 (2014).

[41] D. Leykam and Y. D. Chong, Edge Solitons in NonlinearPhotonic Topological Insulators, Phys. Rev. Lett. 117, 143901 (2016).

[42] M. J. Ablowitz and J. T. Cole, Tight-Binding Methods for General Longitudinally Driven Photonic Lattices: Edge States and Solitons, Phys. Rev. A 96, 043868 (2017).

[43] J. L. Marzuola, M. Rechtsman, B. Osting, and M. Bandres, Bulk Soliton Dynamics in Bosonic Topological Insulators, arXiv:1904.10312.

[44] S. Mukherjee and M. C. Rechtsman, Observation of Floquet Solitons in a Topological Bandgap, Science 368, 856 (2020).

[45] D. Smirnova, D. Leykam, Y. Chong, and Y. Kivshar, Nonlinear Topological Photonics, Appl. Phys. Rev. 7, 021306 (2020).

[46] S. Xia, D. Jukić, N. Wang, D. Smirnova, L. Smirnov, L. Tang, D. Song, A. Szameit, D. Leykam, J. Xu, Z. Chen, and H. Buljan, Nontrivial Coupling of Light into a Defect: The Interplay of Nonlinearity and Topology, Light Sci. Appl. 9, 147 (2020).

[47] L. J. Maczewsky, M. Heinrich, M. Kremer, S. K. Ivanov, M. Ehrhardt, F. Martinez, Y. V. Kartashov, V. V. Konotop, L. Torner, D. Bauer et al., Nonlinearity-Induced Photonic Topological Insulator, Science 370, 701 (2020).

[48] S. Rachel, Interacting Topological Insulators: A Review, Rep. Prog. Phys. 81, 116501 (2018).

[49] Y. S. Kivshar and D. K. Campbell, Peierls-Nabarro Potential Barrier for Highly Localized Nonlinear Modes, Phys. Rev. E 48, 3077 (1993).

[50] Y. S. Kivshar and B. A. Malomed, Dynamics of Solitons in Nearly Integrable Systems, Rev. Mod. Phys. 61, 763 (1989).

[51] O. F. Oxtoby and I. V. Barashenkov, Moving Solitons in the Discrete Nonlinear Schrödinger Equation, Phys. Rev. E 76, 036603 (2007).

[52] P. G. Kevrekidis, The Discrete Nonlinear Schrödinger Equation: Mathematical Analysis, Numerical Computations and Physical Perspectives (Springer Science and Business Media, New York, 2009), Vol. 232.

[53] Note that a tight-binding lattice of helical waveguides in Ref. [41] supports similar perfectly linear edge dispersion.

[54] We note that nonlinearity can also induce coupling from bulk to the edge modes; see Ref. [46]. 
[55] K. M. Davis, K. Miura, N. Sugimoto, and K. Hirao, Writing Waveguides in Glass with a Femtosecond Laser, Opt. Lett. 21, 1729 (1996).

[56] F. Nathan and M.S. Rudner, Topological Singularities and the General Classification of Floquet-Bloch Systems, New J. Phys. 17, 125014 (2015).

[57] R. R. Gattass and E. Mazur, Femtosecond Laser Micromachining in Transparent Materials, Nat. Photonics 2, 219 (2008).
[58] A. Szameit and S. Nolte, Discrete Optics in FemtosecondLaser-Written Photonic Structures, J. Phys. B 43, 163001 (2010).

[59] S. Jensen, The Nonlinear Coherent Coupler, IEEE J. Quantum Electron. 18, 1580 (1982).

[60] See Supplemental Material at http://link.aps.org/ supplemental/10.1103/PhysRevX.11.041057 for Supplemental Movies 1-4. 